\title{
Effects of Bronchodilators on Regional Lung Sound Distribution in Patients with Chronic Obstructive Pulmonary Disease
}

\author{
Masamichi Mineshita $^{a}$ Shin Matsuoka ${ }^{b}$ Teruomi Miyazawa $^{a}$ \\ ${ }^{a}$ Division of Respiratory and Infectious Diseases, Department of Internal Medicine, St. Marianna University School of \\ Medicine, and ${ }^{\text {b } D e p a r t m e n t ~ o f ~ R a d i o l o g y, ~ S t . ~ M a r i a n n a ~ U n i v e r s i t y ~ S c h o o l ~ o f ~ M e d i c i n e, ~ K a w a s a k i, ~ J a p a n ~}$
}

\section{Key Words}

Lung sound · Pulmonary function · Impulse oscillometry

\begin{abstract}
Background: Bronchodilators have been reported to influence regional lung ventilation in patients with chronic obstructive pulmonary disease (COPD), which may change regional lung sound distribution. Vibration response imaging (VRI) is a lung imaging system for the assessment of breath sounds. Objective: To evaluate the effects of a short-acting $\beta_{2}$-agonist (SABA) on the regional distribution of lung sounds in COPD patients. Methods: A double-blind crossover trial was performed to compare the treatment of COPD patients with an SABA ( $20 \mu \mathrm{g}$ of inhaled procaterol) versus a placebo. The percentage of regional lung sound energy [quantitative lung data (QLD)] was evaluated with VRI. VRI, spirometry, and impulse oscillometry (IOS) were performed immediately before and $30 \mathrm{~min}$ after SABA administration. Results: Ten male patients ( $69.6 \pm 14.2$ years of age, percentage predicted forced expiratory volume in $1 \mathrm{~s}: 43.8 \pm 16.9 \%)$ were evaluated. The use of an SABA produced significant functional improvements in the spirometric and IOS measurements. Among the homogeneous emphysema patients $(n=7)$, the upper-lung QLD decreased (from $24.2 \pm 5.8$ to $18.8 \pm 6.1 \%, p<0.05)$ and the lower-lung QLD increased (from $37.9 \pm 12.7$ to $46.1 \pm 14.3 \%$, $p<0.05$ ) following $S A B A$
\end{abstract}

(C) 2013 S. Karger AG, Basel

0025-7931/13/0871-0045\$38.00/0 inhalation. However, the significant redistribution of the regional lung QLD to the lower-lung field was not observed in 2 of the 3 inhomogeneous emphysema patients. Conclusion: The additional use of an SABA by COPD patients improved their pulmonary function, which was accompanied by changes in regional lung air flow. The distribution of emphysematous lesions and the bronchial reactivity to SABA appeared to affect the redistribution of the lung sounds following bronchodilator administration.

Copyright $\odot 2013$ S. Karger AG, Basel

\section{Introduction}

Chronic obstructive pulmonary disease (COPD) is a major cause of chronic morbidity and mortality throughout the world [1]. This disease is characterized by progressive expiratory flow limitation, which leads to incomplete lung emptying and pulmonary hyperinflation, especially during exercise. Pulmonary hyperinflation can cause flattening of the diaphragm, which can impair the piston-like downward displacement capacity of that organ. Symptomatic COPD patients are usually treated with bronchodilators, such as long-acting inhaled anticholinergics and long-acting $\beta_{2}$-agonists [2-4]. Inhaled short-acting $\beta_{2^{-}}$ agonists (SABAs) have a relatively rapid onset of action and are used as a standard method to reduce symptoms in

\section{KARGER}

E-Mail karger@karger.com www.karger.com/res
Teruomi Miyazawa

2-16-1, Sugao

Miyamae-ku

Kawasaki 216-8511 (Japan)

E-Mail miyazawat@marianna-u.ac.jp 
patients with COPD $[5,6]$. These bronchodilators have been reported to produce improvements in exercise tolerance, health-related quality of life, and the exacerbation rate by improving airflow and hyperinflation [7].

Aliverti et al. [8] reported that the use of salbutamol increased forced expiratory volume in $1 \mathrm{~s}\left(\mathrm{FEV}_{1}\right)$, forced vital capacity (FVC), and inspiratory capacity (IC); it also reduced resting functional residual capacity. The salbutamol-induced decrease in the functional residual capacity was accomplished by decreasing the volume of the abdomen rather than that of the total chest wall. These bronchodilator effects appeared to cause a change in regional lung ventilation.

Kirby et al. [9] used magnetic resonance imaging with inhaled hyperpolarized ${ }^{3} \mathrm{He}$ to evaluate regional structurefunction measurements in COPD subjects, and they reported a change in gas distribution following bronchodilator administration. Kirby et al. suggested that the newly ventilated lung regions were not more emphysematous than the lung regions of interest that participated in ventilation prior to bronchodilator administration. Furthermore, they concluded that the bronchodilator use caused a reduction in regional gas trapping. The elucidation of the relationship between regional lung structure and regional ventilation following bronchodilator administration in COPD patients, in whom the distribution of emphysematous or bronchial lesions is thought to vary, may provide new insights for evaluating the clinical benefits of bronchodilators for individual patients. However, the use of magnetic resonance imaging with inhaled hyperpolarized ${ }^{3} \mathrm{He}$ is limited by high costs and poor accessibility.

Airflow in the lung vibrates the airway wall and produces breath sounds. A correlation between breath sound recordings and the regional distribution of pulmonary ventilation has been reported, particularly in studies comparing acoustic findings with data obtained with radioactive gases in normal subjects $[10,11]$. Regional lung sounds may be influenced by improvements in regional lung ventilation induced by bronchodilator administration. Vibration response imaging (VRI) is a commercially available acoustic lung imaging system that displays breath sound energy distribution as a dynamic grey-scale image [12-15]. VRI is a noninvasive, radiation-free technique that requires minimal patient effort and can be transported to the bedside [16, 17]. We recently reported that the locations of central airway obstructions and the outcomes of procedures could be reliably identified by analyzing the specific patterns of lung images obtained with the use of VRI [18]. Additionally, reports of the application of VRI in the diagnosis of obstructive pulmonary diseases have been published [19-21].
We postulated that SABA-induced changes in regional lung ventilation would alter regional lung sound distributions, which would be influenced by lung architecture or bronchial responsiveness to SABAs among COPD patients. We conducted a pilot study to examine the effects of an SABA on pulmonary function and the accompanying changes in lung sound distribution using VRI in patients with moderate-to-severe COPD.

\section{Methods}

\section{Subjects}

We recruited 11 patients who had been previously diagnosed with moderate-to-severe COPD according to the guidelines of the Global Initiative for Chronic Obstructive Lung Disease (GOLD). All the patients were ex-smokers, and the inclusion criteria were a postbronchodilator $\mathrm{FEV}_{1} / \mathrm{FVC}$ ratio of $<0.7$ and a predicted $\mathrm{FEV}_{1}$ of $<80 \%$. The predicted values of the spirometric measurements were derived from the guidelines of the Japanese Respiratory Society [22]. Patients were excluded from the study if they had received a clinical diagnosis of asthma or bronchiectasis. The ethics committee of the St. Marianna University School of Medicine approved this study, and written informed consent was obtained from all the participants. This study is registered with the University Hospital Medical Information Network (UMIN-CTR, No. UMIN000003174).

\section{Study Design}

The study was conducted as a double-blind, randomized, crossover trial and was performed at a single center (St. Marianna University Hospital, Kanagawa, Japan). The patients received either a single 20- $\mu$ g dose of inhaled procaterol (an inhaled SABA, Otsuka Pharmaceutical Co., Ltd., Tokyo, Japan) or a placebo on each of the two study days, which were separated by 4 weeks. A pharmacist advised the patients on how to use the inhaler devices on each study day. The patients were permitted to continue using longacting bronchodilators, such as tiotropium, salmeterol, transdermal turobuterol or theophylline, throughout the duration of the study. All of the tests were performed at approximately the same time of day. The tests were performed in the following order: impulse oscillometry (IOS), VRI, and spirometry. The patients underwent a 6-min walking test (6MWT) at the end of each study day.

\section{Pulmonary Function Tests}

Spirometry was performed with a calibrated spirometer (HI801, Chest M.I., Inc., Tokyo, Japan). Prior to testing, calibration checks were performed using a 3-liter calibration syringe, with ambient air to ensure correct equipment function. Spirometry was performed before and $30 \mathrm{~min}$ after inhaler use according to the

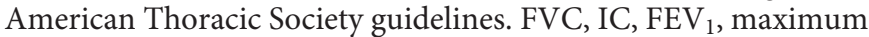
expiratory flow rate at $50 \%$ of FVC, and maximum expiratory flow rate at $25 \%$ of FVC were recorded. For IOS (Masterscreen IOS; Erich Jaeger, Hoechberg, Germany), the subjects supported their cheeks, while impulses were applied during tidal breathing. The average inspiratory and expiratory respiratory resistance $(\mathrm{R})$ and reactance $(\mathrm{X})$ values were calculated at $5 \mathrm{~Hz}$ ( $\mathrm{R} 5$, R5ins, $\mathrm{R} 5 \mathrm{exp}$, $\mathrm{X} 5$, X5ins, and X5exp). Additionally, the respiratory resistance at 
$20 \mathrm{~Hz}$ (R20) and the resonant frequency were recorded. The IOS measurements were performed in triplicate and the mean IOS values were used for further analysis.

VRI Recordings

The VRIxp System (Deep Breeze, Ltd., Or Akiva, Israel) is a computer-based acoustic lung imaging platform that was developed to acquire, quantify, monitor, and store breath sounds. Breath sounds were recorded using the VRI device as previously described $[12,13]$ with 7-row arrays; 6-row arrays were used if the height of the subject was less than $165 \mathrm{~cm}$. Each subject was seated in a quiet environment with their hands resting in their laps. The right and left planar arrays were placed symmetrically on the subject's back using a low-vacuum, computer-controlled method. The two bottom-row arrays were positioned at approximately the same height as the two arrays that were parallel to the vertebral column. The recording was performed over a 12 -second period, while the subject took deep, regular breaths at a rate of 15-20 breaths per minute. VRI software (Deep Breeze) was used to calculate the regional quantitative lung data (QLD). The breathing of each subject was recorded at least 3 times, and the VRI recording with the highest technical quality was chosen for evaluation.

\section{High-Resolution Computed Tomography}

All of the patients were scanned with a 64-detector CT scanner (Aquilion 64, Toshiba Medical Systems, Otawara, Japan). The CT was conducted during a deep inspiration breath-hold with the patient in the supine position. Prior to the CT examination, each patient was carefully instructed on how to breathe during the examination. The CT parameters for the inspiratory scans were as follows: collimation $0.5 \mathrm{~mm}, 120 \mathrm{kVp}, 200 \mathrm{~mA}$, gantry rotation time $0.5 \mathrm{~s}$, and beam pitch 0.83 (table feed per gantry 53 and collimation beam width 64). All images were reconstructed using a standard algorithm, with a slice thickness of $1 \mathrm{~mm}$ and a reconstruction interval of $0.5 \mathrm{~mm}$.

The CT images were transferred to a personal computer for the quantitative analysis of emphysema. Three CT slices were selected from each series of CT scans for each subject. The upper cranial slice was obtained $1 \mathrm{~cm}$ above the upper margin of the aortic arch, the middle slice was obtained $1 \mathrm{~cm}$ below the carina, and the lower caudal slice was obtained $1 \mathrm{~cm}$ below the right inferior pulmonary vein. These CT images were then analyzed using a semiautomatic image processing program (Image J version $1.40 \mathrm{~g}$, a public domain Java image-processing program available at http://rsb.info.nih.gov/ij/). This program uses a semiautomatic threshold technique to isolate the lungs from other tissues and structures, and it selects all of the pixels between -500 and $-1,024 \mathrm{HU}$. Minimal user intervention was required to exclude the nonlung structures that satisfied the threshold criteria, such as the trachea, blood vessels, and large bronchi near the hilum. Low-attenuation areas (LAA) between -950 and $-1,024 \mathrm{HU}$ were then identified as emphysema in the upper-, middle-, and lower-lung fields. A threshold value of -950 HU was selected because this threshold has been macroscopically and microscopically validated for thin-section CT studies of the extent of emphysema [23]. The percentage of the LAA that was less than -950 HU for the entire lung area (\%LAA) was calculated for both the right and left sides of the upper-, middle-, and lower-lung fields as follows: \%LAA $=(\mathrm{LAA} /$ each whole-lung area $) \times 100(\%)$. We defined emphysema-dominant COPD as an averaged regional \%LAA of more than $25 \%$, and we defined heterogeneous emphysema as a greater than $25 \%$ difference between the \%LAAs of three lung fields.

Effects of Bronchodilators on Lung Sound Distribution in COPD Patients
Table 1. Characteristics of subjects $(n=10)$

\begin{tabular}{lc}
\hline Males/females & $10 / 0$ \\
Age, years & $69.6 \pm 14.2$ \\
$\quad$ Range & $32-80$ \\
Body mass index & $22.1 \pm 3.1$ \\
Smoking status & ex-smoker \\
$\quad$ Smoking history, pack-years & $68.9 \pm 37.0$ \\
Baseline lung function & \\
FVC, \% predicted & $80.9 \pm 17.2$ \\
FEV, \% predicted & $43.8 \pm 16.9$ \\
Ratio of FEV ${ }_{1} /$ FVC & $42.0 \pm 9.1$ \\
GOLD stage & \\
II & 5 \\
III & 4 \\
IV & 1 \\
Medication for COPD & \\
Long-acting inhaled anticholinergics & 10 \\
Long-acting $\beta_{2}$-agonists & 4 \\
$\quad$ Inhaled & 2 \\
$\quad$ Transdermal & 2 \\
Inhaled corticosteroids & \\
Theophylline compound & 0 \\
Supplemental oxygen & 3 \\
\hline
\end{tabular}

Values represent number or mean \pm SD.

\section{Statistical Analysis}

The data are expressed as means \pm standard deviation. The measurements of IOS, spirometry, and regional lung QLD before and after the inhalation of procaterol or placebo were compared. These paired data were compared using the Wilcoxon $t$ test. $\mathrm{p}<$ 0.05 was considered to be statistically significant.

\section{Results}

Between 13 February 2010 and 11 June 2011, 11 patients were recruited; however, a VRI sensor array could not be attached to 1 patient due to the bony nature of the structure of his back. Thus, statistical analyses were performed for 10 patients. The patient characteristics and pulmonary function data are shown in table 1. Four patients were treated with tiotropium, 2 with tiotropium and salmeterol, 2 with tiotropium and theophylline, 1 with tiotropium and transdermal tulobuterol, and 1 with tiotropium, transdermal tulobuterol and theophylline. Two patients were prescribed an inhaled SABA for use as required. One of these patients needed an inhaled SABA every morning (07:00), whereas the other patient could forgo the inhaled SABA for more than $8 \mathrm{~h}$ before the administration of the study medication.

No adverse events were reported after the inhalation of the study drugs. The placebo had no effect on the pul- 
Table 2. Procaterol effects on regional lung airflow

\begin{tabular}{|c|c|c|c|c|}
\hline & \multicolumn{2}{|l|}{ Placebo } & \multicolumn{2}{|l|}{$\underline{\text { Procaterol }}$} \\
\hline & before inhalation & after inhalation & before inhalation & after inhalation \\
\hline \multicolumn{5}{|l|}{ Spirometry } \\
\hline FVC, 1 & $2.63 \pm 0.45$ & $2.64 \pm 0.44$ & $2.66 \pm 0.58$ & $2.80 \pm 0.58$ \\
\hline IC, 1 & $1.82 \pm 0.42$ & $1.81 \pm 0.06$ & $1.75 \pm 0.43$ & $1.89 \pm 0.44^{*}$ \\
\hline $\mathrm{FEV}_{1}, \mathrm{l}$ & $1.12 \pm 0.35$ & $1.12 \pm 0.31$ & $1.15 \pm 0.37$ & $1.25 \pm 0.40^{*}$ \\
\hline MEFR50\% & $0.47 \pm 0.21$ & $0.47 \pm 0.19$ & $0.48 \pm 0.21$ & $0.55 \pm 0.23 *$ \\
\hline MEFR25\% & $0.20 \pm 0.08$ & $0.18 \pm 0.06$ & $0.22 \pm 0.10$ & $0.23 \pm 0.10$ \\
\hline \multicolumn{5}{|l|}{ IOS } \\
\hline $\mathrm{R} 5, \mathrm{kPa} / \mathrm{l} / \mathrm{s}$ & $0.37 \pm 0.10$ & $0.40 \pm 0.12$ & $0.38 \pm 0.10$ & $0.31 \pm 0.10^{*}$ \\
\hline R5insp & $0.33 \pm 0.08$ & $0.32 \pm 0.09$ & $0.31 \pm 0.10$ & $0.27 \pm 0.08^{*}$ \\
\hline $\mathrm{R} 5 \exp$ & $0.40 \pm 0.12$ & $0.44 \pm 0.15$ & $0.42 \pm 0.12$ & $0.33 \pm 0.11^{* *}$ \\
\hline $\mathrm{R} 20, \mathrm{kPa} / \mathrm{l} / \mathrm{s}$ & $0.23 \pm 0.05$ & $0.24 \pm 0.06$ & $0.24 \pm 0.08$ & $0.20 \pm 0.04^{*}$ \\
\hline $\mathrm{R} 5-\mathrm{R} 20, \mathrm{kPa} / \mathrm{l} / \mathrm{s}$ & $0.14 \pm 0.08$ & $0.16 \pm 0.09$ & $0.13 \pm 0.07$ & $0.11 \pm 0.08^{*}$ \\
\hline $\mathrm{X} 5, \mathrm{kPa} / \mathrm{l} / \mathrm{s}$ & $-0.19 \pm 0.08$ & $-0.21 \pm 0.11$ & $-0.19 \pm 0.10$ & $-0.17 \pm 0.13$ \\
\hline X5insp & $-0.16 \pm 0.04$ & $-0.16 \pm 0.05$ & $-0.15 \pm 0.06$ & $-0.13 \pm 0.06^{*}$ \\
\hline $\mathrm{X} 5 \exp$ & $-0.22 \pm 0.12$ & $-0.26 \pm 0.20$ & $-0.21 \pm 0.15$ & $-0.22 \pm 0.23$ \\
\hline Resonant frequency, $\mathrm{Hz}$ & $23.4 \pm 5.9$ & $24.2 \pm 5.6$ & $22.4 \pm 5.9$ & $19.9 \pm 6.5^{* *}$ \\
\hline \multicolumn{5}{|l|}{$Q L D, \%$} \\
\hline \multicolumn{5}{|l|}{ Right } \\
\hline Upper & $10.5 \pm 4.0$ & $11.2 \pm 4.7$ & $12.8 \pm 4.0$ & $9.8 \pm 5.2^{*}$ \\
\hline Middle & $16.4 \pm 6.5$ & $16.6 \pm 4.9$ & $20.3 \pm 6.3$ & $16.5 \pm 6.4$ \\
\hline Lower & $17.7 \pm 11.3$ & $16.9 \pm 9.1$ & $20.5 \pm 12.0$ & $18.6 \pm 10.6$ \\
\hline \multicolumn{5}{|l|}{ Left } \\
\hline Upper & $14.9 \pm 7.9$ & $13.1 \pm 4.7$ & $11.8 \pm 4.0$ & $12.5 \pm 5.6$ \\
\hline Middle & $21.1 \pm 5.3$ & $20.8 \pm 6.8$ & $16.9 \pm 5.4$ & $18.4 \pm 5.0$ \\
\hline Lower & $19.4 \pm 7.8$ & $21.3 \pm 7.6$ & $17.7 \pm 10.3$ & $24.2 \pm 11.8^{*}$ \\
\hline
\end{tabular}

Continuous valuables before and after inhalation were tested with Wilcoxon's t test. Values are represented as means \pm standard deviation. ${ }^{*} \mathrm{p}<0.05,{ }^{* *} \mathrm{p}<0.01$. MEFR $50 \%=$ Maximum expiratory flow rate at $50 \%$ of FVC; MEFR25\% = maximum expiratory flow rate at 25\% of FVC; R20 = respiratory resistance at $20 \mathrm{~Hz}$; X = respiratory reactance; insp = values measured during inspiration; exp = values measured during expiration.

monary function tests or regional lung QLD (table 2). Procaterol use significantly increased IC, $\mathrm{FEV}_{1}$ and maximum expiratory flow rate at $50 \%$ of $\mathrm{FVC}$, according to the spirometry results, and produced significant changes in the IOS parameters, excluding the $\mathrm{X} 5$ and $\mathrm{X} 5 \mathrm{exp}$ values (table 2). There were no significant differences in the $6 \mathrm{MWT}$ distances between the procaterol and placebo groups (procaterol: $430 \pm 56 \mathrm{~m}$, placebo: $428 \pm 59 \mathrm{~m}$ ). Although the right upper-lung QLD was significantly reduced (from $12.8 \pm 4.0$ to $9.8 \pm 5.2 \%, \mathrm{p}<0.05$ ), and the left lower-lung QLD was significantly increased (from $17.7 \pm 10.3$ to $24.2 \pm 11.8 \%, \mathrm{p}<0.05)$ after procaterol inhalation (table 2), there were no significant changes in the total upper, middle, or lower QLDs.

To study the effects of structural changes in the lungs on lung sound distribution, we compared the distribution of emphysematous lesions as indicated by the chest high- resolution CT (HRCT) results and the procaterol effects on the regional lung QLD (table 3). Among the patients with homogeneous emphysema $(n=7)$, the total QLD of the upper lung was significantly decreased after procaterol inhalation, whereas the total QLD of the lower lung was significantly increased (fig. 1; upper-lung QLD: from $24.2 \pm 5.8$ to $18.8 \pm 6.1 \%, \mathrm{p}<0.05$; lower-lung QLD: from $37.9 \pm 12.7$ to $46.1 \pm 14.3 \%, \mathrm{p}<0.05)$. Figure 2 shows the HRCT findings and the VRI recordings for case 3 . This subject was a 77-year-old male with stage 2 COPD (predicted $\mathrm{FEV}_{1}=51.5 \%$, predicted $\left.\mathrm{FVC}=91.9 \%\right)$ and an average \%LAA of $51.5 \%$. Procaterol inhalation decreased the upper-lung QLD and increased the lower-lung QLD. In case 4 (upper-lung-dominant emphysema) and case 6 (non-emphysema-dominant COPD with a higher \%LAA in the lower-lung field), the regional lung sound energy shifted from the emphysematous field to a more pre- 
Fig. 1. Among the patients with homogeneous emphysema $(n=7)$, the total QLD of the upper lung was significantly decreased after procaterol inhalation, whereas the total QLD of the lower lung was significantly increased (upper-lung QLD: from $24.2 \pm$ 5.8 to $18.8 \pm 6.1 \%, \mathrm{p}<0.05$; lower-lung QLD: from $37.9 \pm 12.7$ to $46.1 \pm 14.3 \%$, p < 0.05).

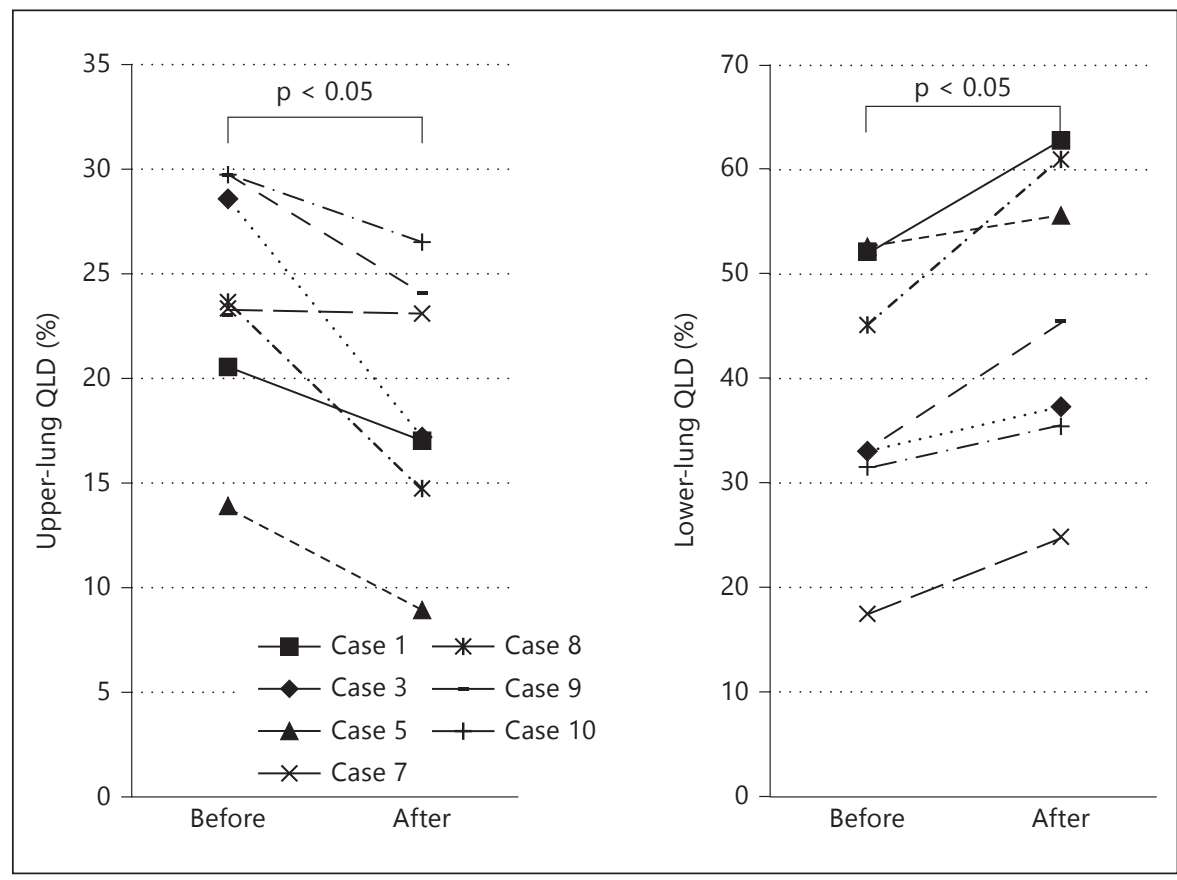

Table 3. Distribution of emphysematous lesions on chest HRCT and procaterol effects on regional lung QLD

\begin{tabular}{|c|c|c|c|c|c|c|c|}
\hline & \multicolumn{4}{|l|}{$\%$ LAA } & \multicolumn{3}{|c|}{ Change in QLD, $\%^{\mathrm{a}}$} \\
\hline & upper & middle & lower & phenotype & upper & middle & lower \\
\hline Case 1 & 77.6 & 79.8 & 68.3 & hom-E & -3.55 & -7.22 & 10.78 \\
\hline Case 2 & 59.0 & 42.0 & 23.6 & het-E & 8.30 & 4.55 & -12.87 \\
\hline Case 3 & 57.9 & 44.9 & 51.8 & hom-E & -11.38 & 6.98 & 4.40 \\
\hline Case 4 & 58.0 & 42.0 & 31.6 & het-E & -3.30 & -3.97 & 7.25 \\
\hline Case 5 & 36.5 & 27.7 & 33.2 & hom-E & -4.94 & 1.94 & 3.00 \\
\hline Case 6 & 8.7 & 7.6 & 20.6 & non-E & 10.82 & -4.84 & -5.98 \\
\hline Case 7 & 57.8 & 52.2 & 52.0 & hom-E & -0.29 & -7.10 & 7.39 \\
\hline Case 8 & 37.1 & 33.2 & 19.4 & hom-E & -8.93 & -6.93 & 15.86 \\
\hline Case 9 & 22.9 & 21.1 & 33.9 & hom-E & -5.63 & -6.79 & 12.40 \\
\hline Case 10 & 69.5 & 68.1 & 65.2 & hom-E & -3.23 & -0.69 & 3.90 \\
\hline
\end{tabular}

hom-E = Homogeneous emphysema; het-E = heterogeneous emphysema; non-E = emphysema nondominant.

${ }^{a}$ QLD of after inhalation - QLD of before inhalation.

served lung field (upper to lower in case 4 and lower to upper in case 6). However, the QLD of the most emphysematous fields increased after bronchodilator use in case 2. Figure 3 shows the HRCT and VRI data for case 2, a 73-year-old male with stage $3 \mathrm{COPD}$ (predicted $\mathrm{FEV}_{1}=$ $33.0 \%$, predicted $\mathrm{FVC}=65.1 \%$ ) and an average \%LAA of $41.6 \%$. His bilateral upper-lung QLD was increased after procaterol inhalation, whereas the bilateral lower-lung QLD was decreased.

Effects of Bronchodilators on Lung Sound Distribution in COPD Patients

\section{Discussion}

This is the first study to simultaneously evaluate the effects of bronchodilator use on pulmonary function and regional lung sound distribution in patients with COPD. We found that an SABA (procaterol) caused improvements in pulmonary function that were accompanied by changes in regional lung sound distribution in moderateto-severe COPD patients, whereas the placebo caused no 


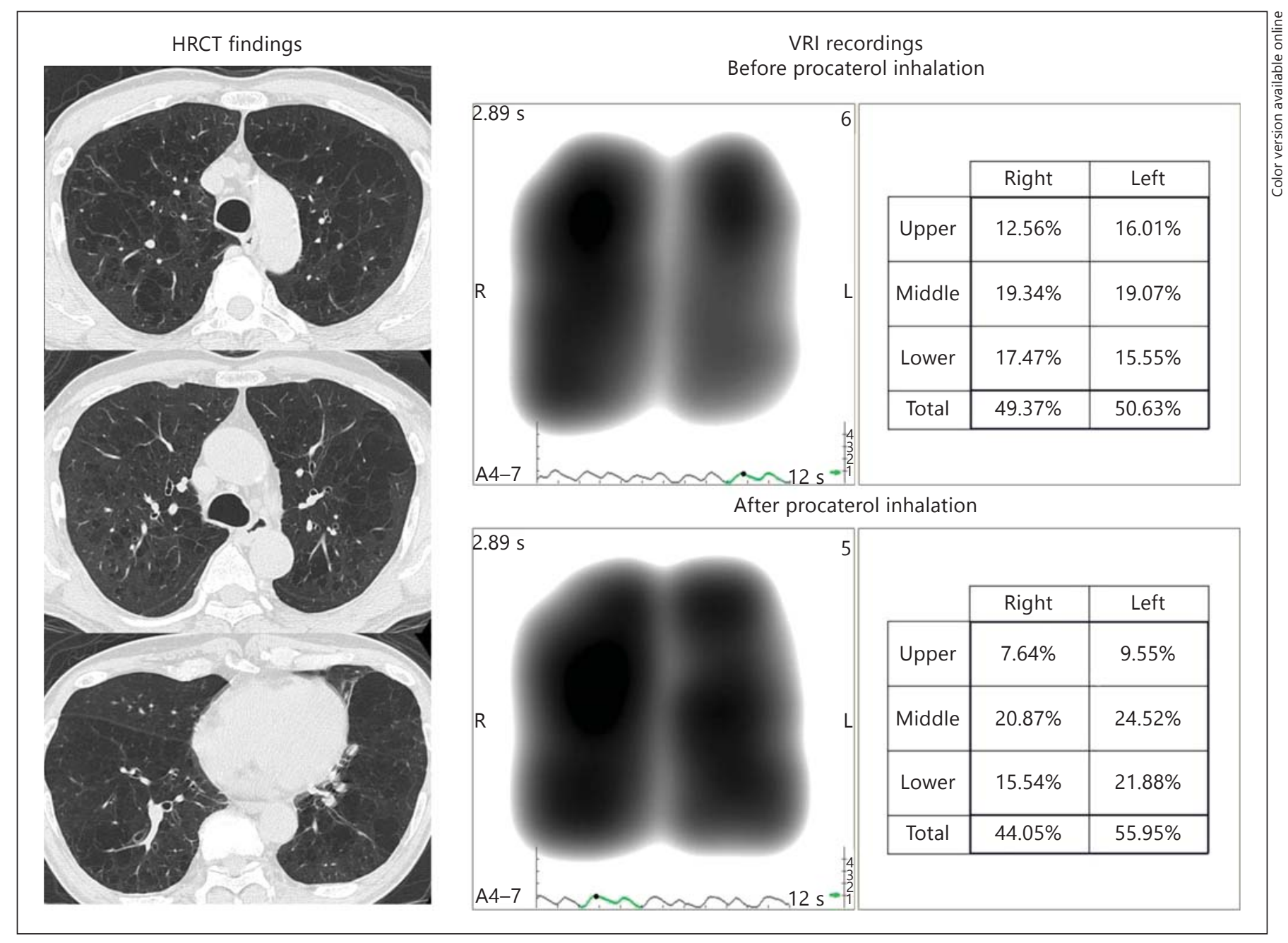

Fig. 2. HRCT and VRI data for case 3, a 77-year-old male with stage $2 \mathrm{COPD}$ (predicted $\mathrm{FEV}_{1}=51.5 \%$, predicted $\mathrm{FVC}=91.9 \%$ ) with homogeneous emphysema and an average \%LAA of 51.5\%. Procaterol inhalation decreased the upper-lung QLD and increased the lower-lung QLD.

significant changes. However, the patterns of change in lung sound distribution after bronchodilator use were different between COPD patients with homogeneous emphysema and other types of COPD patients.

In homogeneous emphysema, the redistribution of vibration energy to lower-lung regions after bronchodilator use may be a surrogate marker of improved diaphragm movement. In inhomogeneous emphysema, the relationship between regional lung structure and the airflow shift after bronchodilator use may vary between patients. Hatipoğlu et al. [24] reported that an SABA (albuterol) relieved dyspnea and enhanced respiratory muscle output, which could be explained by a lengthening of the diaphragm due to decreased lung volume. Bronchodila- tor administration appears to improve diaphragm movement by deflating the lungs, which may lead to increased ventilation of the lower-lung field and an airflow shift to that field. Dellinger et al. [16] reported the redistribution of vibration energy to lower-lung regions in pressure-targeted modes of mechanical ventilation and speculated that the physiological mechanism might involve an increase in the downward movement of the diaphragm. Therefore, prior to this study, we expected that the pulmonary function improvements caused by bronchodilators might be accompanied by incremental changes in lower-lung breath sound energy. Indeed, we observed this relationship in patients with homogeneous emphysema. In 7 homogeneous patients, the regional lung QLD, 


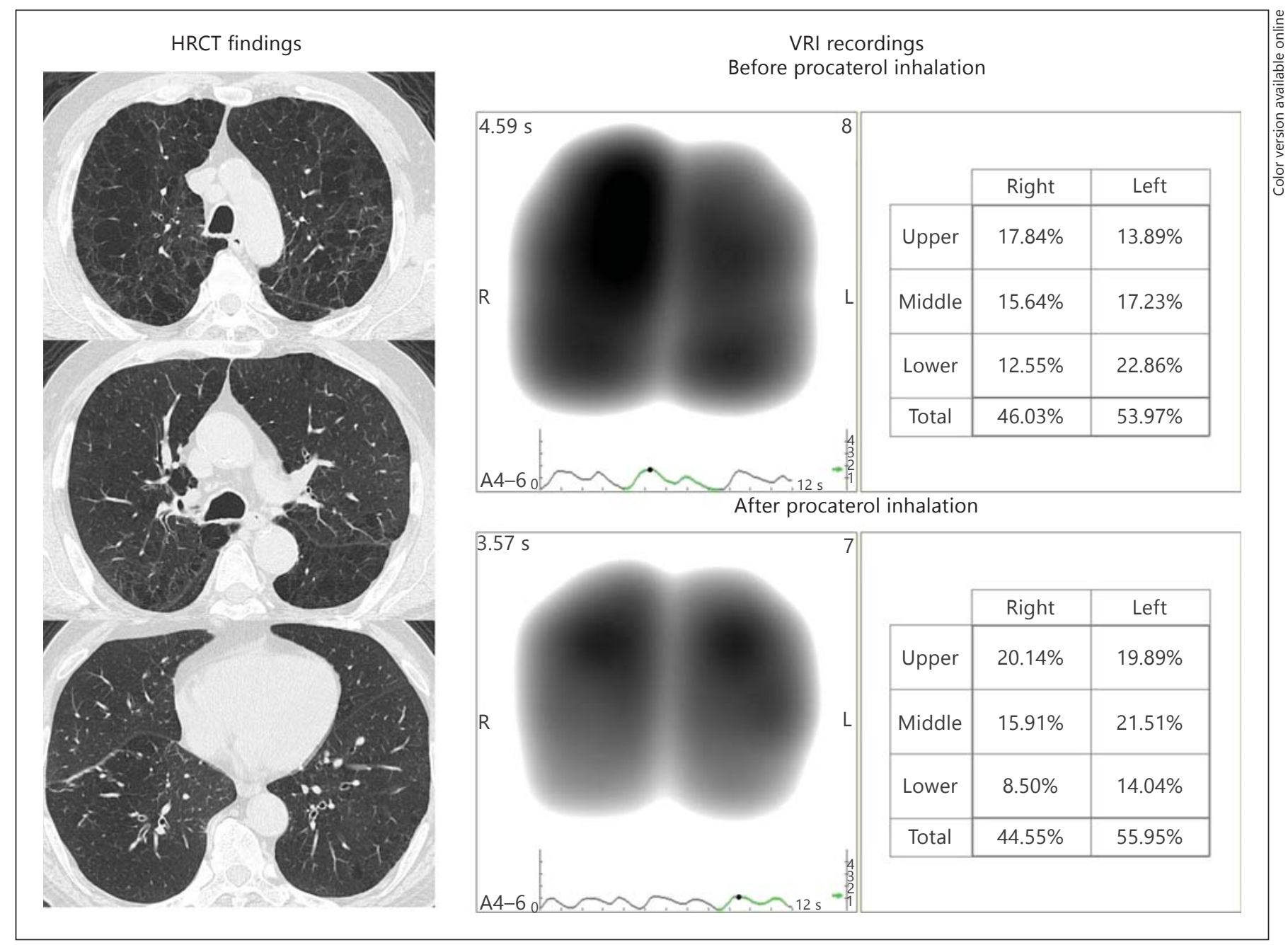

Fig. 3. HRCT and VRI data for case 2, a 73-year-old male with stage 3 COPD (predicted $\mathrm{FEV}_{1}=33.0 \%$, predicted FVC $=65.1 \%$ ) with nonhomogeneous emphysema and an average \%LAA of 41.6\%. His bilateral upper-lung QLD was increased after procaterol inhalation, whereas his bilateral lower-lung QLD was decreased.

which was considered a possible surrogate marker for regional lung airflow, was decreased in the upper-lung field and increased in the lower-lung field after SABA inhalation. In patients with homogeneous emphysema, analyzing lung sound distributions may be useful for assessing diaphragm movement after COPD treatment.

We considered the changes in lung sound distribution following bronchodilator administration that were observed in the patients with heterogeneous emphysema and nonemphysema COPD to be influenced by heterogeneities in the lung structure, such as differences in the distribution of emphysematous lesions or in the reactivity of the bronchus to inhaled bronchodilators. In COPD, bronchodilators are delivered primarily to the more nor- mal lung regions [9]. This effect is beneficial for ventilation-perfusion matching. In this study, we found that the regional lung QLD of the more preserved lung regions was increased in one case of upper-lung-dominant emphysema and in one case of non-emphysema-dominant COPD. In these cases, the bronchodilator was thought to cause an airflow shift to the more normal lung field, and the increase in the regional QLD appeared to reflect this phenomenon.

However, we observed one case of heterogeneous emphysema in which the regional lung QLD of the relatively emphysematous upper lung increased after bronchodilator inhalation. In this case, the bronchodilator use actually changed the regional lung sound distribution, albeit 
in an apparently discordant manner. If the increase in regional lung QLD reflected an airflow shift to the more damaged part of the lung, the ventilation-perfusion mismatch of this case may have been worsened. Not all COPD patients benefit from bronchodilator therapy, and there is only a weak relationship between the changes in spirometric parameters and the subsequent exercise capacity [25]. However, this patient experienced an improvement in the 6MWT distance (459-473 m), with a lower drop in $\mathrm{SpO}_{2}$ levels during the 6MWT (from 10 to $7 \%$ ) following procaterol inhalation. Therefore, a worsening of his ventilation-perfusion mismatch appeared to be unlikely.

One possible mechanism of bronchodilator activity in this case is that in bronchi dilated by SABA, the turbulence caused by airflow may decrease, which reduces the regional lung sound intensity. Conversely, the bronchi of more diseased lungs may not dilate sufficiently after SABA inhalation without a change in the regional lung sound intensity. As a result, the relative intensity of the lung sounds (QLD) throughout the diseased lung field is increased. Because this theory is based on speculation alone, further studies using quantitative CT or radioactive gases are required to find out the reason for this phenomenon. The relationship between regional lung structure and changes in regional lung sounds after bronchodilator use may vary, especially among heterogeneous or nonemphysematous COPD patients.

In this study, the use of long-acting bronchodilators was continued to avoid aggravating the patients' conditions. We also investigated whether the use of SABA really produced genuine physiological effects on the daily management of moderate-to-severe COPD. Because the effect of SABAs on spirometry is thought to be ameliorated by the regular use of long-acting bronchodilators, we added IOS as a more sensitive assessment to elucidate the physiological effects of an SABA $[26,27]$. We found significant improvements using both IOS and spirometric assessments.

Although this was a pilot study with a small number of participants, we discovered changes in lung sound distribution accompanied by pulmonary function improvements following bronchodilator use by COPD patients. The redistribution of vibration energy to the lower-lung regions after treatment may be a surrogate marker for improved diaphragm movement, especially among homogeneous emphysema patients. Larger studies are needed to elucidate the relationships among lung architecture, the bronchial response to treatment, and changes in regional lung sound distribution.

In conclusion, the addition of SABA treatment in moderate-to-severe COPD patients improved pulmonary function, which was accompanied by a change in regional lung airflow. In homogeneous emphysema patients, the redistribution of vibration energy to lowerlung regions after bronchodilator administration may be a surrogate marker for the deflation of lungs accompanied by an improvement in the diaphragm movement. In inhomogeneous emphysema, the relationship between regional lung structure and the airflow shift after bronchodilator use may vary between patients.

\section{Acknowledgements}

The authors would like to thank Mr. Jason Tonge for his assistance in the preparation of the manuscript and Hiroko Minatogawa and Tsutomu Watanabe for their pharmacological assistance.

\section{Financial Disclosure and Conflicts of Interest}

The VRI equipment was provided by Deep Breeze, Ltd. This work was supported by JSPS KAKENHI grant No. 24591143.

\section{References}

1 The Global Strategy for the Diagnosis, Management and Prevention of COPD, Global Initiative for Chronic Obstructive Lung Disease (GOLD) 2010. http://www.goldcopd.org.

$>2$ Rennard SI, Anderson W, ZuWallack R, Broughton J, Bailey W, Friedman M, Wisniewski M, Rickard K: Use of a long-acting inhaled $\beta_{2}$-adrenergic agonist, salmeterol xinafoate, in patients with chronic obstructive pulmonary disease. Am J Respir Crit Care Med 2001;163:1087-1092.

3 Tashkin DP, Celli B, Senn S, Burkhart D, Kesten S, Menjoge S, Decramer MA: 4-year trial of tiotropium in chronic obstructive pulmonary disease. N Engl J Med 2008;359:15431554.

4 Donohue JF, Fogarty C, Lötvall J, Mahler DA, Worth H, Yorgancioglu A, Iqbal A, Swales J, Owen R, Higgins M, Kramer B: Once-daily bronchodilators for chronic obstructive pulmonary disease. Indacaterol versus tiotropium. Am J Respir Crit Care Med 2010;182: 155-162.

5 Belman MJ, Botnick WC, Shin JW: Inhaled bronchodilators reduce dynamic hyperinflation during exercise in patients with chronic obstructive pulmonary disease. Am J Respir Crit Care Med 1996;153:967-975.

-6 Cook D, Guyatt G, Wong E, Goldstein R, Bedard M, Austin P, Ramsdale H, Jaeschke R, Sears M: Regular versus as-needed short-acting inhaled $\beta$-agonist therapy for chronic obstructive pulmonary disease. Am J Respir Crit Care Med 2001;163:85-90.

$>7$ Deesomchok A, Webb KA, Forkert L, Lam YM, Ofir D, Jensen D, O’Donnell DE: Lung hyperinflation and its reversibility in patients with airway obstruction of varying severity. COPD 2010;7:428-437. 
-8 Aliverti A, Rodger K, Dellacà RL, Stevenson N, Lo Mauro A, Pedotti A, Calverley PM: Effect of salbutamol on lung function and chest wall volumes at rest and during exercise in COPD. Thorax 2005;60:916-924.

-9 Kirby M, Heydarian M, Wheatley A, McCormack DG, Parraga G: Evaluating bronchodilator effects in chronic obstructive pulmonary disease using diffusion-weighted hyperpolarized helium-3 magnetic resonance imaging. J Appl Physiol 2012;112:651-657.

10 Ploy-Song-Sang Y, Martin RR, Ross WR, Loudon RG, Macklem PT: Breath sounds and regional ventilation. Am Rev Respir Dis 1977; 116:187-199.

-11 Ploy-Song-Sang Y, Macklem PT, Ross WR: Distribution of regional ventilation measured by breath sounds. Am Rev Respir Dis 1978; 117:657-664.

-12 Dellinger RP, Parrillo JE, Kushnir A, Rossi M, Kushnir I: Dynamic visualization of lung sounds with a vibration response device: a case series. Respiration 2008;75:60-72.

-13 Maher TM, Gat M, Allen D, Devaraj A, Wells AU, Geddes DM: Reproducibility of dynamically represented acoustic lung images from healthy individuals. Thorax 2008; 63:542-548.

14 Anantham D, Herth FJ, Majid A, Michaud G, Ernst A: Vibration response imaging in the detection of pleural effusions: a feasibility study. Respiration 2009;77:166-172.
15 Blanco M, Mor R, Fraticelli A, Breen DP, Dutau H: Distribution of breath sound images in patients with pneumothoraces compared to healthy subjects. Diagnostic yield of vibration response imaging technology. Respiration 2009;77:173-178.

16 Dellinger RP, Jean S, Cinel I, Tay C, Rajanala S, Glickman YA, Parrillo JE: Regional distribution of acoustic-based lung vibration as a function of mechanical ventilation mode. Crit Care 2007;11:R26.

17 Lev S, Glickman YA, Kagan I, Shapiro M, Moreh-Rahav O, Dahan D, Cohen J, Grinev M, Singer P: Computerized lung acoustic monitoring can help to differentiate between various chest radiographic densities in critically ill patients. Respiration 2010; 80:509-516.

18 Becker HD, Slawik M, Miyazawa T, Gat M: Vibration response imaging as a new tool for interventional-bronchoscopy outcome assessment: a prospective pilot study. Respiration 2009;77:179-194.

19 Guntupalli KK, Reddy RM, Loutfi RH, Alapat PM, Bandi VD, Hanania NA: Evaluation of obstructive lung disease with vibration response imaging. J Asthma 2008;45:923-930.

20 Wang Z, Bartter T, Baumann BM, Abouzgheib W, Chansky ME, Jean S: Asynchrony between left and right lung in acute asthma. J Asthma 2008;45:575-578.

21 Wang Z, Jean S, Bartter T: Lung sound analysis in the diagnosis of obstructive airway disease. Respiration 2009;77:134-138.
22 The Committee of Pulmonary Physiology, the Japanese Respiratory Society: Guidelines for pulmonary function tests: spirometry, flowvolume curve, diffusion capacity of the lung (in Japanese). Tokyo, Japanese Respiratory Society, 2004

23 Gevenois PA, De Vuyst P, de Maertelaer V, Zanen J, Jacobovitz D, Cosio MG, Yernault JC: Comparison of computed density and microscopic morphometry in pulmonary emphysema. Am J Respir Crit Care Med 1996; 154:187-192.

24 Hatipoğlu U, Laghi F, Tobin MJ: Does inhaled albuterol improve diaphragmatic contractility in patients with chronic obstructive pulmonary disease? Am J Respir Crit Care Med 1999; 160:1916-1921.

25 Hay JG, Stone P, Carter J: Bronchodilator reversibility, exercise performance and breathlessness in stable chronic obstructive pulmonary disease. Eur Respir J 1992;5:659-664.

26 Borrill ZL, Houghton CM, Tal-Singer R, Vessey SR, Faiferman I, Langley SJ, Singh D: The use of plethysmography and oscillometry to compare long-acting bronchodilators in patients with COPD. Br J Clin Pharmacol 2008;65:244-252.

27 Williamson PA, Short PM, Clearie KL, Vaidyanathan S, Fardon TC, Howaniec LJ, Lipworth BJ: Paradoxical trough effects of triple therapy with budesonide/formoterol and tiotropium bromide on pulmonary function outcomes in COPD. Chest 2010;138:595-604. 